EESTI NSV TEADUSTE AKADEEMIA TOIMETISED. XII KOIDE FOOSIKA-MATEMAATIKA- JA TEHNIKATEADUSTE SEERIA. 1963, NR. 4

ИЗВЕСТИЯ АКАДЕМИИ НАУК ЭСТОНСКОИ ССР. ТОМ ХІІ

СЕРИЯ ФИЗИКО-МАТЕМАТИЧЕСКИХ И ТЕХНИЧЕСКИХ НАУК. 1963, 스 4

\title{
ОБ ИТЕРАТИВНЫХ МЕТОДАХ РЕШЕНИЯ НЕЛИНЕЙНОГО УРАВНЕНИЯ, ОСНОВАННОГО НА ЛИНЕАРИЗАЦИИ ПРИ ПОМОЩИ ИНТЕРПОЛЯЦИОННОИ ФОРМУЛЫ НЬЮТОНА
}

\author{
с. ульм,
}

кандидат физико-математических наук

1. М. А. Красносельский и Я. Б. Рутицкий доказали $\left[{ }^{3}\right]$ некоторые общие теоремы о сходимости приближенных методов решения нелинейного операторного уравнения

$$
P(x)=0,
$$

основанных на линеаризации уравнения (1) при помощи формульі Тэйлора.

В данной статье мы исследуем вопрос о сходимости приближенных методов решения уравнения (1), основанных на линеаризации при помощи интерполяционной формулы Ньютона. В дальнейшем допустим, что оператор $P$ действует из банахового пространства $X$ в линейное нормированное пространство $У$.

Пусть $x_{n-1}$ и $x_{n}$ некоторые приближения к решению $x^{*}$ уравнения (1). Вместо уравнения (1) рассмотрим линеаризованное уравнение

$$
P\left(x_{n}\right)+P\left(x_{n}, x_{n-1}\right)\left(x-x_{n}\right)=0,
$$

где $P\left(x_{n}, x_{n-1}\right)$ - аналог разделенных разностей первого порядка для оператора $P(x)[4,6]$.

Пусть для приближенного решения линейного уравнения

$$
A x=b
$$

имеется некоторый метод

$$
\bar{x}_{1}=V\left(\bar{x}_{0} ; A, b\right),
$$

где оператор $V$ в общем нелинейный; $\overline{x_{0}}$ - начальное приближение $к$. решению $\bar{x}$ уравнения (3), а элемент $\bar{x}_{1}$ аппроксимирует точное решение $\bar{x}$ в некотором смысле лучше, чем $\bar{x}_{0}$.

Если метод (4) применять последовательно для приближенного решения линеаризованных уравнений (2), мы получим для решения уравнения (1), исходя из начальных приближений $x_{0}$ и $x_{-1}$, следующий итеративный метод:

$$
\begin{gathered}
x_{n+1}=V\left(x_{n} ; P\left(x_{n}, x_{n-1}\right), P\left(x_{n}, x_{n-1}\right) x_{n}-P\left(x_{n}\right)\right) \\
(n=0,1,2, \ldots) .
\end{gathered}
$$


Установим нижеследующие условия:

$1^{\circ}$ для метода (4) известна оценка погрешности в виде

$$
\left\|\bar{x}_{1}-\bar{x}\right\| \leqslant q\left\|\bar{x}_{0}-\bar{x}\right\|, q<1,
$$

которая применима для уравнения (2).

$2^{\circ}$ для каждого $x^{\prime}, x^{\prime \prime}, x^{\prime \prime \prime}$ из некоторой замкнутой сферы $S$ существуют $P\left(x^{\prime \prime}, x^{\prime \prime \prime}\right),\left[P\left(x^{\prime}, x^{\prime \prime}\right)\right]^{-1}=\Lambda\left(x^{\prime}, x^{\prime \prime}\right)$ и справедлива оценка

$$
\left\|E-\Lambda\left(x^{\prime}, x^{\prime \prime}\right) P\left(x^{\prime \prime}, x^{\prime \prime \prime}\right)\right\| \leqslant K\left\|x^{\prime}-x^{\prime \prime \prime}\right\|,
$$

где $E$ - тождественный оператор пространства $X$.

$3^{\circ}$ оператор $P(x)$ и $\Lambda\left(x^{\prime}, x^{\prime \prime}\right)$ являются непрерывными в сфере $S$.

$4^{\circ} \quad\left\|\Lambda\left(x_{0}, x_{-1}\right) P\left(x_{0}\right)\right\| \leqslant \eta_{0}$;

$5^{\circ} \quad\left\|x_{0}-x_{-1}\right\| \leqslant(1+q) \gamma$.

Введем обозначения:

$$
\begin{gathered}
\eta_{n}=\left\|\Lambda\left(x_{n}, x_{n-1}\right) P\left(x_{n}\right)\right\| \\
d_{0}=q+K(1+q)\left(\eta_{0}+\gamma\right) ; \\
d_{n}=q+K(1+q)\left(\eta_{n}+\eta_{n-1}\right) \quad(n=1,2, \ldots) \\
d=\max \left\{d_{0} ; d_{1}\right\} ; \\
D=1+\sum_{k=0}^{\infty}\left(\prod_{i=0}^{k} d_{i}\right)=1+d_{0}+d_{0} d_{1}+d_{0} d_{1} d_{2}+\ldots
\end{gathered}
$$

Те орем а 1: Если выполнены условия $1^{\circ}-5^{\circ}$, причем в качестве $S$ выбрана сфера

$$
\left\|x-x_{0}\right\| \leqslant(1+q) \cdot \max \left\{\gamma ; D \eta_{0}\right\}
$$

$u d<1$, то уравнение (1) имеет в сфере (15) единственное решение $x^{*}$, к которому последовательность (5) сходится со скоростью

$$
\left\|x^{*}-x_{n}\right\| \leqslant(1+q) \eta_{0} \cdot \sum_{k=n-1}^{\infty}\left(\prod_{i=0}^{k} d_{i}\right)<\frac{1+q}{1-d} d^{n} \eta_{0}
$$

где $n=1,2, \ldots$.

Д ока зательство: Обозначим точные решения уравнений через $x_{n}^{*}(n=0,1, \ldots)$.

Используя условия $1^{\circ}$ и $4^{\circ}$, получим

$$
\begin{aligned}
& \left\|x_{1}-x_{0}\right\| \leqslant\left\|x_{1}-x_{0}^{*}\right\|+\left\|x_{0}^{*}-x_{0}\right\| \leqslant q\left\|x_{0}^{*}-x_{0}\right\|+\left\|x_{0}^{*}-x_{0}\right\| \leqslant \\
& \leqslant(1+q)\left\|x_{0}^{*}-x_{0}\right\|=(1+q)\left\|\Lambda\left(x_{0}, x_{-1}\right) P\left(x_{0}\right)\right\|=(1+q) \eta_{0} .
\end{aligned}
$$

На основании определения аналога разделенных разностей [4]

$$
\begin{gathered}
P\left(x_{1}\right)=P\left(x_{0}\right)+P\left(x_{1}, x_{0}\right)\left(x_{1}-x_{0}\right)+P\left(x_{0}, x_{-1}\right)\left(x_{0}^{*}-x_{0}\right)- \\
-P\left(x_{0}, x_{-1}\right)\left(x_{0}^{*}-x_{0}\right)= \\
=P\left(x_{1}, x_{0}\right)\left(x_{1}-x_{0}^{*}\right)+\left[P\left(x_{1}, x_{0}\right)-P\left(x_{0}, x_{-1}\right)\right]\left(x_{0}^{*}-x_{0}\right) .
\end{gathered}
$$


Поскольку теперь

$$
\Lambda\left(x_{1}, x_{0}\right) P\left(x_{1}\right)=x_{1}-x_{0}^{*}+\left[E-\Lambda\left(x_{1}, x_{0}\right) P\left(x_{0}, x_{-1}\right)\right]\left(x_{0}^{*}-x_{0}\right),
$$

то используя условия $1^{\circ}, 2^{\circ}, 4^{\circ}$ и $5^{\circ}$ и оценку (17), получим

$$
\begin{aligned}
& \eta_{1} \leqslant q\left\|x_{0}-x_{0}^{*}\right\|+K\left\|x_{1}-x_{-1}\right\|\left\|x_{0}-x_{0}^{*}\right\| \leqslant \\
& \leqslant\left[q+K\left(\left\|x_{1}-x_{0}\right\|+\left\|x_{0}-x_{-1}\right\|\right)\right]\left\|x_{0}-x_{0}^{*}\right\| \leqslant \\
& \leqslant\left[q+K(1+q)\left(\eta_{0}+\gamma\right)\right] \eta_{0}=d_{0} \eta_{0} .
\end{aligned}
$$

Вообще получим для $n=1,2, \ldots$

$$
\begin{gathered}
\left\|x_{n+1}-x_{n}\right\| \leqslant\left\|x_{n+1}-x_{n}^{*}\right\|+\left\|x_{n}^{*}-x_{n}\right\| \leqslant \\
\leqslant q\left\|x_{n}^{*}-x_{n}\right\|+\left\|x_{n}^{*}-x_{n}\right\| \leqslant \\
\leqslant(1+q)\left\|x_{n}^{*}-x_{n}\right\|=(1+q)\left\|\Lambda\left(x_{n}, x_{n-1}\right) P\left(x_{n}\right)\right\|=(1+q) \eta_{n} ; \\
P\left(x_{n+1}\right)=P\left(x_{n}\right)+P\left(x_{n+1}, x_{n}\right)\left(x_{n+1}-x_{n}\right)+ \\
+P\left(x_{n}, x_{n-1}\right)\left(x_{n}^{*}-x_{n}\right)-P\left(x_{n}, x_{n-1}\right)\left(x_{n}^{*}-x_{n}\right)= \\
=P\left(x_{n+1}, x_{n}\right)\left(x_{n+1}-x_{n}^{*}\right)+\left[P\left(x_{n+1}, x_{n}\right)-P\left(x_{n}, x_{n-1}\right)\right]\left(x_{n}^{*}-x_{n}\right) \\
\Lambda\left(x_{n+1}, x_{n}\right) P\left(x_{n+1}\right)=x_{n+1}-x_{n}^{*}+\left[E-\Lambda\left(x_{n+1}, x_{n}\right) P\left(x_{n}, x_{n-1}\right)\right]\left(x_{n}^{*}-x_{n}\right) \\
\quad \vdots \\
\eta_{n+1} \leqslant q\left\|x_{n}^{*}-x_{n}\right\|+K\left\|x_{n+1}-x_{n-1}\right\|\left\|x_{n}^{*}-x_{n}\right\| \leqslant \\
\leqslant\left[q+K\left(\left\|x_{n+1}-x_{n}\right\|_{*}^{*}+\left\|x_{n}-x_{n-1}\right\|\right)\right]\left\|x_{n}^{*}-x_{n}\right\| \leqslant \\
\leqslant\left[q+K(1+q)\left(\eta_{n}+\eta_{n-1}\right)\right] \eta_{n}=d_{n} \eta_{n}
\end{gathered}
$$

Так как по условию теоремы

$$
d=\max \left\{d_{0} ; d_{1}\right\}<1,
$$

то поочередно для $n=2,3, \ldots$ получим, что

$d_{n}=q+K(1+q)\left(\eta_{n}+\eta_{n-1}\right)<q+K(1+q)\left(\eta_{1}+\eta_{0}\right)=d_{1}<1$

и $\eta_{n+1}<\eta_{n}$.

Итак, справедливы соотношения:

$$
\begin{aligned}
& \eta_{1} \leqslant d_{0} \eta_{0} \leqslant d \eta_{0} \\
& \eta_{2} \leqslant d_{1} \eta_{1} \leqslant d_{0} d_{1} \eta_{0} \leqslant d^{2} \eta_{0} \\
& \quad \ldots \ldots \\
& \eta_{n+1} \leqslant d_{n} \eta_{n} \leqslant d_{0} d_{1} \ldots d_{n} \eta_{0}<d^{n+1} \eta_{0} .
\end{aligned}
$$

Так как

$$
\begin{gathered}
\left\|x_{n+p}-x_{n}\right\| \leqslant\left\|x_{n+p}-x_{n+p-1}\right\|+\ldots+\left\|x_{n+1}-x_{n}\right\| \leqslant \\
\leqslant(1+q)\left(\eta_{n+p-1}+\ldots+\eta_{n}\right) \leqslant \\
\leqslant(1+q) d_{0} d_{1} \ldots d_{n-1}\left(1+d_{n}+d_{n} d_{n-1}+\ldots+d_{n} \ldots d_{n+p-2}\right) \eta_{0} \leqslant \\
\leqslant(1+q) d^{n}\left(1+d+d^{2}+\ldots+d^{p-1}\right) \eta_{0},
\end{gathered}
$$

то последовательность (5) является фундаментальной. Итак, существует $\lim x_{n}=x^{*}$. Переходя к пределу $(p \rightarrow \infty)$ в неравенствах $(25)$ полу$n \rightarrow \infty$

чим оценки (16). 
Покажем, что $x^{*}$ является решением уравнения (1). Поскольку

$$
\lim _{n \rightarrow \infty} \eta_{n}=\lim _{n \rightarrow \infty}\left\|\Lambda\left(x_{n}, x_{n-1}\right) P\left(x_{n}\right)\right\|=0,
$$

то $\Lambda\left(x^{*}, x^{*}\right) P\left(x^{*}\right)=0$. Применив слева к последнему равенству оператор $P\left(x^{*}, x^{*}\right)=P^{\prime}\left(x^{*}\right)$, мы получим: $P\left(x^{*}\right)=0$, т. е. $x^{*}$ является решеннем уравнения (1).

Выше мы использовали принадлежность элементов $x_{-1}, x_{0}, \ldots$, $x_{n}, \ldots, x^{*}$ к сфере (15). Это обстоятельство вытекает из неравенств:

$$
\begin{gathered}
\left\|x_{-1}-x_{0}\right\| \leqslant(1+q) \gamma ; \\
\left\|x_{n}-x_{0}\right\| \leqslant\left\|x_{n}-x_{n-1}\right\|+\ldots+\left\|x_{1}-x_{0}\right\| \leqslant \\
\leqslant(1+q)\left(\eta_{n-1}+\ldots+\eta_{0}\right) \leqslant \\
\leqslant(1+q)\left(1+d_{0}+d_{0} d_{1}+\ldots+d_{0} d_{1} \ldots d_{n-1}\right) \eta_{0} \leqslant(1+q) D \eta_{0} .
\end{gathered}
$$

Переходя к пределу $(n \rightarrow \infty)$ в (29) убедимся, что и $x^{*} \in S$.

Покажем единственность решения уравнения (1) в сфере (16). Допустив, что в сфере (16) существует два различных решений $x^{*}$ и $x^{* *}$, получим:

$$
0=P\left(x^{*}\right)-P\left(x^{* *}\right)=P\left(x^{*}, x^{* *}\right)\left(x^{*}-x^{* *}\right) .
$$

Применив слева к последнему равенству оператор $\Lambda\left(x^{*}, x^{* *}\right)$, получим противоречие: $x^{*}=x^{* *}$.

Теорема деказана.

Рассмотрим случай, когда условие $1^{\circ}$ заменено условием $1^{\circ}$ : для метода (4) известна оценка погрешности в виде

$$
\left\|\bar{x}_{1}-\bar{x}\right\| \leqslant \varrho\left\|\bar{x}_{1}-\bar{x}_{0}\right\|, \varrho<\frac{1}{2},
$$

которая применима для уравнений (2), и условие $5^{\circ}$ условием $5^{\circ}$ :

$$
\left\|x_{0}-x_{-1}\right\| \leqslant \frac{\gamma}{1-0} .
$$

Введем обозначения:

$$
\begin{gathered}
\delta_{0}=\frac{\varrho+K\left(\eta_{0}+\vartheta\right)}{1-\varrho} ; \\
\delta_{n}=\frac{\varrho+K\left(\eta_{n}+\eta_{n-1}\right)}{1-\varrho} \quad(n=1,2, \ldots) ; \\
\delta=\max \left\{\delta_{0}, \delta_{1}\right\} ; \\
\Delta=1+\sum_{k=0}^{\infty}\left(\prod_{i=0}^{k} \delta_{i}\right)=1+\delta_{0}+\delta_{0} \delta_{1}+\delta_{0} \delta_{1} \delta_{2}+\ldots
\end{gathered}
$$

Т еорем а 2: Если выполнены условия $1^{\circ \prime}, 2^{\circ}, 3^{\circ}, 4^{\circ}$ и $5^{\circ}$, причем в качестве $S$ выбрана сфера

$$
\left\|x-x_{0}\right\| \leqslant(1-\mathrm{e})^{-1} \cdot \max \left\{\gamma ; \Delta \eta_{0}\right\}
$$

и $\delta<1$, то уравнение (1) имеет в сфере (38) единственное решение $x^{*}$, $\kappa$ которому последовательность (5) сходится со скоростью: 


$$
\left\|x^{*}-x_{n}\right\| \leqslant \frac{\eta_{0}}{1-\varrho} \sum_{k=n-1}^{\infty}\left(\prod_{i=0}^{k} \delta_{i}\right)<\frac{\delta^{n}}{(1-\varrho)(1-\delta)} \eta_{0},
$$

где $n=1,2, \ldots$.

Доказательство: Используя условия $1^{\circ}$ и $4^{\circ}$, получим

$$
\left\|x_{1}-x_{0}\right\| \leqslant\left\|x_{1}-x_{0}^{*}\right\|+\left\|x_{0}^{*}-x_{0}\right\| \leqslant \mathrm{Q}\left\|x_{1}-x_{0}\right\|+\eta_{0},
$$
откуда

$$
\left\|x_{1}-x_{0}\right\| \leqslant \frac{\eta_{0}}{1-0} .
$$

На основании полученной оценки и формулы (19)

$$
\begin{gathered}
\eta_{1}=\left\|\Lambda\left(x_{1}, x_{0}\right) P\left(x_{1}\right)\right\| \leqslant \varrho\left\|x_{1}-x_{0}\right\|+K\left\|x_{1}-x_{-1}\right\|\left\|x_{0}^{*}-x_{0}\right\| \leqslant \\
\leqslant \frac{\varrho}{1-\delta} \eta_{0}+K\left(\left\|x_{1}-x_{0}\right\|+\left\|x_{0}-x_{-1}\right\|\right)\left\|x_{0}^{*}-x_{0}\right\| \leqslant \\
\leqslant \frac{\varrho+K\left(\eta_{0}+\gamma\right)}{1-\varrho} \eta_{0}=\delta_{0} \eta_{0} .
\end{gathered}
$$

Вообще получим для $n=1,2, \ldots$, оценки

и

$$
\left\|x_{n+1}-x_{n}\right\| \leqslant \frac{\eta_{n}}{1-0}
$$

$$
\eta_{n+1}=\left\|\Lambda\left(x_{n+1}, x_{n}\right) P\left(x_{n+1}\right)\right\| \leqslant \delta_{n} \eta_{n} .
$$

Теперь легко убедиться в правильности нашей теоремы аналогично доказательству теоремы 1 .

3 а меч ан и е 1: При решении уравнения (3) методами класса (4) часто потребуется от оператора $A$ самосопряженность. В связи с этим отметим, что теоремы 1 и 2 остаются справедливыми, если вместо уравнений (2) рассматривать уравнения

$$
P^{*}\left(x_{n}, x_{n-1}\right) P\left(x_{n}\right)+P^{*}\left(x_{n}, x_{n-1}\right) P\left(x_{n}, x_{n-1}\right)\left(x-x_{n}\right)=0
$$

с самосопряженными операторами $P^{*}\left(x_{n}, x_{n-1}\right) P\left(x_{n}, x_{n-1}\right)$, причем через $P^{*}\left(x_{n}, x_{n-1}\right)$ обозначен оператор, сопряженный к линейному оператору $P\left(x_{n}, x_{n-1}\right)$.

Метод (5) имеет в этом случае вид:

$$
\begin{gathered}
x_{n+1}=V\left(x_{n} ; P^{*}\left(x_{n}, x_{n-1}\right) P\left(x_{n}, x_{n-1}\right) ; P^{*}\left(x_{n}, x_{n-1}\right) P\left(x_{n}, x_{n-1}\right) x_{n}-\right. \\
\left.-P^{*}\left(x_{n}, x_{n-1}\right) P\left(x_{n}\right)\right) \\
(n=0,1,2, \ldots) .
\end{gathered}
$$

3 амечание 2: Теоремы 1 и 2 остаются в силе, если условие 2。 заменить условием $2^{\circ}$ : для каждого $x^{\prime}, x^{\prime \prime}, x^{\prime \prime \prime}$ из замкнутой сферы $S$ справедлива оценка

$$
\left\|\Lambda\left(x^{\prime}, x^{\prime \prime}\right) P\left(x^{\prime}, x^{\prime \prime}, x^{\prime \prime \prime}\right)\right\| \leqslant K,
$$

где $P\left(x^{\prime}, x^{\prime \prime}, x^{\prime \prime \prime}\right)$ - аналог разделенных разностей второго порядка оператора $P(x)$ (см. [4, 5]). 
Действительно, если выполнено $2^{\circ \prime}$, то

$$
\begin{gathered}
\left\|E-\Lambda\left(x^{\prime}, x^{\prime \prime}\right) P\left(x^{\prime \prime}, x^{\prime \prime \prime}\right)\right\|=\left\|\Lambda\left(x^{\prime}, x^{\prime \prime}\right)\left[P\left(x^{\prime}, x^{\prime \prime}\right)-P\left(x^{\prime \prime}, x^{\prime \prime \prime}\right)\right]\right\|= \\
=\left\|\Lambda\left(x^{\prime}, x^{\prime \prime}\right) P\left(x^{\prime}, x^{\prime \prime}, x^{\prime \prime \prime}\right)\left(x^{\prime}-x^{\prime \prime \prime}\right)\right\| \leqslant \\
\leqslant\left\|\Lambda\left(x^{\prime}, x^{\prime \prime}\right) P\left(x^{\prime}, x^{\prime \prime}, x^{\prime \prime \prime}\right)\right\|\left\|x^{\prime}-x^{\prime \prime \prime}\right\| \leqslant K\left\|x^{\prime}-x^{\prime \prime \prime}\right\|,
\end{gathered}
$$

т. е. выполнено и условие $2^{\circ}$.

В качестве примера рассмотрим применение метода наискорейшего спуска ['] для решения уравнений $\left(2^{\prime}\right)$. Метод $\left(5^{\prime}\right)$ в этом случае принимает вид

$$
\begin{gathered}
x_{n+1}=x_{n}-\frac{\left\|P^{*}\left(x_{n}, x_{n-1}\right) P\left(x_{n}\right)\right\|^{2}}{\left\|P\left(x_{n}, x_{n-1}\right) P^{*}\left(x_{n}, x_{n-1}\right) P\left(x_{n}\right)\right\|^{2}} P^{*}\left(x_{n}, x_{n-1}\right) P\left(x_{n}\right) \\
(n=0,1, \ldots)
\end{gathered}
$$

т. е. совпадает с методом минимальных невязок в классе итерационных методов $x_{n+1}=x_{n}-\varepsilon_{n} P^{*}\left(x_{n}, x_{n-1}\right) P\left(x_{n}\right)$ (см. ['] $)$.

Пусть для каждого $h \in X$ и $x^{\prime}, x^{\prime \prime}, x^{\prime \prime \prime} \in S$ справедливы оценки

$$
\begin{gathered}
\frac{1}{\sqrt{M}}\|\dot{h}\| \leqslant\left\|P\left(x^{\prime}, x^{\prime \prime}\right) h\right\| \leqslant \sqrt{K}\|h\|, \\
\left\|P\left(x^{\prime}, x^{\prime \prime}\right)-P\left(x^{\prime \prime}, x^{\prime \prime \prime}\right)\right\| \leqslant L\left\|x^{\prime}-x^{\prime \prime \prime}\right\|, \\
\left\|\Lambda\left(x_{0}, x_{-1}\right) P\left(x_{0}\right)\right\| \leqslant \eta_{0}, \\
\left\|x_{0}-x_{-1}\right\| \leqslant(1+q) \gamma .
\end{gathered}
$$

Применяя теорему 1 , можно взять $\left[{ }^{2}\right]$

$$
q=\frac{1-\mu}{t_{0}+\mu^{3}} \sqrt{\frac{t_{0}\left(t_{0}+\mu^{4}\right)}{t_{0}+1}}
$$

где

$$
\mu=\frac{1}{M K}
$$

и $t_{0}-$ положительный корень уравнения

$$
t^{3}-\mu^{3}(1-2 \mu) t^{2}-\mu^{3}(2-\mu) t-\mu^{7}=0 .
$$

На основании теоремы 1 условие сходимости метода (45) выражается в виде

$$
\max \left\{d_{0}, q+\sqrt{M} L(1+q)\left(1+d_{0}\right) \eta_{0}\right\}<1,
$$

где

$$
d_{0}=q+\sqrt{M} L(1+q)\left(\eta_{0}+\gamma\right) .
$$

Условие (53) несколько более ограничивающее, чем соответствующее условие в теореме, полученной прямым путем (см. [6], теорема 5), но зато не требуется знания оценки снизу для $\left\|P^{*}\left(x^{\prime}, x^{\prime \prime}\right) h\right\|$.

\section{Л И Т Е Р Т У Р А}

1. Л. В. К а н торо в и ч, Функциональный анализ и прикладная математика, УМН, 1948, вып. $6(28), 3,89-185$.

2. В. Н. Қоста ч ук и Б. П. Пугачев, Точная оценка уменьшения погрешности на одном шаге метода наискорейшего спуска, Тр. Семинара по функц. анализу Воронежского ун-та, 1956, вып. 2, 25-30.

3 ENSV TA Toimetised T-4 63 
3. М. А. К раснисельский и Я. Б. Рутицкий, О некоторых приближенных методах решения нелинейных операторных уравнений, основанных на линеарнзацин, ДАН, 1961, 141, 785-788.

4. А. С. С е рге е в, О методе хорд, Сибирскнй матем. журнал, 1961, 2, 2, 282-289.

5. С. У льм, О6 ннтерполяцнонных методах решения нелинейных уравнений в пространстве Банаха, Изв. АН ЭССР. Сер. физ.-матем. и техн. наук, 1963, 1, 24-30.

6. С. У льм, Об одном классе итерационных методов в пространстве Гильберта, Изв. АН ЭССР. Сер. физ.-матем. н техн. наук, 1963, 2, 132-140.

Ннститут кибернетики

Академии наук Эстонской ССР

Поступила в редакцию 27. IV 1963

\title{
NEWTONI INTERPOLATSIOONIVALEMIGA LINEARISEERIMISEL. POHINEVATEST MITTELINEAARSE VORRANDI LAHENDAMISE ITERATSIOONIMEETODITEST
}

\author{
S. Ulm, \\ füsika-matemaatikateaduste kandidaat
}

\section{Resümee}

Vaadeldakse mittelineaarse operaatorvõrrandi (1) lahenidamise iteratsioonimeetodeid, mis saadakse igal sammul võrrandi (1) lineariseerimisel Newtoni interpolatsioonivalemiga, kusjuures saadud (lineaarsed) vôrrandid lahendatakse mingi meetodiga ligikaudselt. Tôestatakse kaks ūldist koonduvusteoreemi ja tuuakse näide saadud teoreemide rakendamisest.

Eesti NSV Teaduste Akadeemia Küberneetika Instituut
Saabus toimetusse

27. IV 1963

\section{UBER ITERATIONSMETHODEN FUR DIE LOSUNG NICHTLINEARER GLEICHUNG, WELCHE AUF DER LINEARISIERUNG MITTELS DER NEWTONSCHEN INTERPOLATIONSFORMEL FUSSEN}

\section{S. Ulm}

\section{Zusammenfassung}

Es werden Iterationsmethoden für die angenäherte Lösung der Operatorgleichung (1) untersucht, die allenfalls auf der Linearisierung der Gleichung (1) mittels der Newtonschen Interpolationsformel und, weiter, auf der Anwendung angenäherter Methoden für die Lösung linearer Operatorgleichungen fussen. Es werden zwei allgemeine Konvergenztheoreme abgeleitet und ein Beispiel ihrer Anwendung betrachtet. 